\title{
CONTROL QUÍMICO DE MALEZA DE HOJA ANCHA EN SORGO PARA GRANO
}

\author{
CHEMICAL CONTROL OF BROADLEAF WEEDS IN GRAIN SORGHUM
}

\author{
Enrique Rosales-Robles ${ }^{1 \star}$, Ricardo Sánchez-de la Cruz ${ }^{1}$ y Pedro A. Cerda-García ${ }^{2}$
}

\begin{abstract}
${ }^{1}$ Campo Experimental Río Bravo, Instituto Nacional de Investigaciones Agrícolas, Forestales y Pecuarias (INIFAP). Carretera Matamoros-Reynosa Km. 61. 88900, Río Bravo, Tamaulipas, México. Tel. (899) 934-1045. ${ }^{2}$ Departamento de Parasitología Agrícola, Universidad Autónoma Agraria Antonio Narro. 25315, Buenavista, Saltillo, Coahuila, México.

*Autor para correspondencia (rosales.enrique@inifap.gob.mx)
\end{abstract}

\section{RESUMEN}

El control ineficiente de malezas es un problema importante en la producción de sorgo para grano (Sorghum bicolor L. Moench) en el norte de Tamaulipas, México. En 2007 y 2008 se evaluaron en campo algunas opciones para el control químico de malezas de hoja ancha en sorgo en pre- (PRE) y post- emergencia (POST). Atrazina y amicarbazone a $750 \mathrm{~g} \mathrm{ha}^{-1}$ aplicados PRE resultaron un buen control de polocote (Helianthus annuus L.) y quelite (Amaranthus palmeri S. Wats), sin causar daños al sorgo. En los herbicidas POST aplicados en sorgo de seis hojas, prosulfuron a $17 \mathrm{~g} \mathrm{ha}^{-1}$ mostró un buen control de polocote en ambos años, pero el control de quelite fue medio en 2008 y en ningún año controló al trompillo (Solanum elaeagnifolium Cav.) La mezcla de carfentrazone $2.5 \mathrm{~g} \mathrm{ha}^{-1}+2,4-\mathrm{D} 225 \mathrm{~g} \mathrm{ha}^{-1}$ y 2,4-D a 720 $\mathrm{g} \mathrm{ha}^{-1}$ mostraron buen control de maleza, aunque causaron daños al sorgo y redujeron su rendimiento. Dicamba a $144 \mathrm{~g} \mathrm{ha}^{-1}$ se comportó en forma similar al 2,4-D en control de maleza, pero causó menores daños al sorgo y no disminuyó su rendimiento. Bromoxinil a $480 \mathrm{~g} \mathrm{ha}^{-1}$ controló eficientemente al polocote y al quelite, pero no tuvo efectos sobre trompillo. Bentazona a $960 \mathrm{~g} \mathrm{ha}^{-1}$ tuvo un control regular de polocote, pero no fue eficiente en quelite y trompillo. Los resultados indican que hay varias opciones para el control de polocote y quelite, y que para lograr el control de trompillo se debe incluir un herbicida hormonal como 2,4-D o dicamba.

Palabras clave: Helianthus annuus, Amaranthus palmeri, Solanum elaeagnifolium, herbicidas, sorgo.

\section{SUMMARY}

Ineffective weed control is an important problem in grain sorghum (Sorghum bicolor L. Moench) production in northern Tamaulipas, México. Several options for chemical weed control in sorghum in pre-(PRE) and post - emergence (POST) were evaluated under field conditions in 2007 and 2008. Atrazine and amicarbazone $750 \mathrm{~g} \mathrm{ha}^{-1}$ applied PRE resulted in good control of wild sunflower (Helianthus annuus L.) and Palmer amaranth (Amaranthus palmeri S. Wats), without causing damage to sorghum plants. Among herbicides applied POST to six-leaves sorghum, prosulfuron $17 \mathrm{~g} \mathrm{ha}^{-1}$ showed excellent control of wild sunflower in both years, however Palmer amaranth control was only regular in 2008 and it did not control silverleaf nightshade (Solanum elaeagnifolium Cav.) in any year. The mixture of carfentrazone $2.5 \mathrm{~g} \mathrm{ha}^{-1}+2,4-\mathrm{D} 225 \mathrm{~g} \mathrm{ha}^{-1}$ and 2, 4-D to $720 \mathrm{~g} \mathrm{ha}^{-1}$ showed regular weed control, but damage was caused in sorghum reducing grain yield. Dicamba at $144 \mathrm{~g} \mathrm{ha}^{-1}$ resulted in a similar weed control to 2, 4-D but with less damages to sorghum and without reducing its yield. Bromoxinil $480 \mathrm{~g} \mathrm{ha}^{-1}$ effectively controlled wild sunflower and Palmer amaranth but had no effect on silverleaf nightshade. Bentazon at $960 \mathrm{~g} \mathrm{ha}^{-1}$ controlled wild sunflower, but it was ineffective on Palmer amaranth and on silverleaf nightshade. Results indicate that there are several options for the control of wild sunflower and Palmer amaranth, while silverleaf nightshade must be controlled by adding a POST hormonal herbicide such as 2,4 -D or dicamba.

Index words: Helianthus annuus, Amaranthus palmeri, Solanum elaeagnifolium, herbicides, grain sorghum.

\section{INTRODUCCIÓN}

El sorgo para grano (Sorghum bicolor L. Moench) es uno de los cultivos anuales más importantes en México. En 2009 se cultivaron 1.96 millones de hectáreas con una producción de 6.1 millones de toneladas (SAGARPA, 2011). Sin embargo, México es el principal importador de grano de sorgo en el mundo ya que en promedio de 2005 a 2008 se importaron 2.1 millones de toneladas anuales para satisfacer el mercado nacional (Financiera Rural, 2009). Tamaulipas es el estado con mayor producción de sorgo en México, con una superficie cosechada de $941 \mathrm{mil}$ hectáreas y una producción de 2.59 millones de toneladas en 2009, de las cuales la región norte produjo 2.2 millones de toneladas en 712 mil hectáreas en el ciclo otoño-invierno (SAGARPA, 2011). Un factor que limita la producción de sorgo en el norte de Tamaulipas es el control deficiente de malezas de hoja ancha, entre las que destacan el polocote (Helianthus annuus L.), el quelite (Amaranthus palmeri S. Wats), la amargosa (Parthenium hysterophorus L.), el trompillo (Solanum elaeagnifolium Cav.) y la oreja de ratón (Convolvulus arvensis L.) (Rosales-Robles et al., 2005b).

Los productores de sorgo del norte de Tamaulipas realizan de dos a tres escardas para el control de malezas, que complementan con la aplicación de herbicidas y en algunos casos con deshierbes manuales. El 2,4-D es el herbicida más usado para el control selectivo de malezas de hoja ancha en post-emergencia (POST) en esta región. Este herbicida presenta buena selectividad en sorgo cuando la planta tiene un estado de desarrollo de tres a seis hojas, ya que si se usa fuera de esta etapa es común que cause daños al cultivo (Rosales-Robles et al., 2005b). Además, debido 
a los vientos fuertes en esta región los daños a cultivos sensibles por el acarreo de este herbicida son comunes, particularmente en algodonero (Gossypium hirsutum L.) y ocra (Abelmoschus esculentus L. Moench). A pesar de sus riesgos, los productores de sorgo utilizan al 2,4-D por su bajo costo. Por ello es conveniente contar con otras opciones para el control químico de malezas de hoja ancha, con mayor efectividad y selectividad al sorgo. en un paso de arado, dos pasos de rastra y surcado a 80 $\mathrm{cm}$. En ambos años se sembró el híbrido de sorgo 'Pioneer $82 \mathrm{G}^{\prime} 3^{\prime}$ a 250000 plantas ha ${ }^{-1}$ y se fertilizó con la fórmula $120 \mathrm{~N}-40 \mathrm{P}-00 \mathrm{~K}$. Se proporcionó un riego de siembra y dos de auxilio a los 40 y 60 d después de la siembra. Las fechas de siembra, aplicación de herbicidas, y la población y estado de desarrollo de las malezas al momento de la aplicación, se presentan en el Cuadro 1.

Cuadro 1. Fecha de siembra de sorgo, y aplicación de herbicidas post-emergentes, y densidad de población y estado de desarrollo de las malezas. Río Bravo, Tam.

\begin{tabular}{|c|c|c|c|c|c|c|c|c|}
\hline \multirow[t]{2}{*}{ Año } & \multirow[t]{2}{*}{ Siembra } & \multirow[t]{2}{*}{ Aplicación } & \multicolumn{2}{|c|}{ Helianthus annuus } & \multicolumn{2}{|c|}{ Amaranthus palmeri } & \multicolumn{2}{|c|}{ Solanum elaeagnifolium } \\
\hline & & & Núm. m ${ }^{-2}$ & Hojas & Núm. $m^{-2}$ & Hojas & Núm. $\mathrm{m}^{-2}$ & Hojas \\
\hline 2007 & $21-\mathrm{feb}$ & 20-mar & 30 & 4 & 5 & 4 & 5 & 4 \\
\hline 2008 & $25-\mathrm{feb}$ & 24-mar & 177 & 4 & 10 & 6 & 7 & 6 \\
\hline
\end{tabular}

Entre las alternativas posibles al 2,4-D están atrazina, bromoxinil, bentazona, dicamba y prosulfuron. La atrazina es un herbicida pre-emergente (PRE) de acción básica en malezas de hojas ancha, utilizado ampliamente en sorgo (Wicks et al., 1994). Bromoxinil y bentazona son herbicidas POST de contacto, inhibidores del fotosistema II, que también tienen un control efectivo de malezas de hoja ancha (Baumann et al., 1999). Dicamba es un herbicida de la familia de los reguladores de crecimiento o auxínicos, con acción similar al 2,4-D pero con menor toxicidad al sorgo (Wicks et al., 1994). Prosulfuron es un herbicida de la familia de las sulfonilureas que controla bien al polocote y amargosa y que se caracteriza por requerir bajas dosis de aplicación, vida media corta, baja volatilidad y baja solubilidad en agua, lo que minimiza las posibilidades de daño a otros cultivos (Grichar et al., 2000; O'Sullivan y Thomas 2001; Rosales-Robles et al., 2005b). Entre las nuevas opciones en el control químico de maleza de hoja ancha en sorgo está el amicarbazone, herbicida PRE con acción similar a atrazina; y la mezcla de carfentrazone + 2,4-D, que combina la acción de contacto de carfentrazone y la acción sistémica de 2,4-D. El objetivo de este trabajo fue evaluar algunas opciones de herbicidas para el control de maleza de hoja ancha en sorgo.

\section{MATERIALES Y MÉTODOS}

Los experimentos de campo se establecieron en los ciclos otoño-invierno 2007 y 2008 en el Campo Experimental Río Bravo, Tamaulipas, del Instituto Nacional de Investigaciones Forestales, Agrícolas y Pecuarias (27 $57^{\prime}$ $\left.\mathrm{LN}, 98^{\circ} 10^{\prime} \mathrm{LO}\right)$. Los suelos experimentales son de textura arcillosa (24\% arena, $18 \%$ limo y $58 \%$ arcilla) con pH 7.1 y un contenido de materia orgánica de $1.1 \%$. Estos lotes se seleccionaron por contar con una alta población de polocote, la maleza más común y problemática en el norte de Tamaulipas. En ambos años además hubo poblaciones de quelite y trompillo. La preparación del suelo consistió
El diseño experimental fue bloques completos al azar con 10 tratamientos, y cuatro repeticiones, cuya unidad experimental constó de cuatro surcos de $10 \mathrm{~m}$ de largo. La aplicación de los herbicidas se hizo con un aspersor de mochila motorizado con boquillas de abanico plano 8004 con una presión de salida de $275 \mathrm{kPa}$ y un volumen de aspersión equivalente a $250 \mathrm{~L} \mathrm{ha}^{-1}$. Los herbicidas evaluados fueron: amicarbazone $750 \mathrm{~g} \mathrm{ha}^{-1} \mathrm{y}$ atrazina $750 \mathrm{~g} \mathrm{ha}^{-1}$ aplicados PRE un día después de la siembra; prosulfuron a $17 \mathrm{~g} \mathrm{ha}^{-1}$; 2,4-D amina $720 \mathrm{~g} \mathrm{ha}^{-1}$ (referido posteriormente como 2,4-D); carfentrazone a $2.5 \mathrm{~g} \mathrm{ha}^{-1}+2,4-\mathrm{D}$ a $225 \mathrm{~g} \mathrm{ha}^{-1}$; dicamba a $144 \mathrm{~g} \mathrm{ha}^{-1}$; bromoxinil a $480 \mathrm{~g} \mathrm{ha}^{-1} \mathrm{y}$ bentazona a $960 \mathrm{~g} \mathrm{ha}^{-1}$ aplicados POST cuando el sorgo tenía seis hojas liguladas, para determinar con mayor rigor la selectividad al sorgo. Se incluyeron dos tratamientos como referencia para las evaluaciones de fitotoxicidad y control de maleza, un testigo con maleza y un testigo limpio con deshierbes manuales realizados semanalmente durante las primeras ocho semanas de desarrollo del cultivo, periodo crítico de competencia con maleza (Rosales-Robles et al., 2005a).

La fitotoxicidad al sorgo y a la maleza se evaluó mediante estimaciones visuales en una escala de 0 a $100 \%$, donde 0 indica sin efecto y $100 \%$ indica la muerte de la planta (Cuadro 2), escala propuesta por la Sociedad Europea de Investigación en Maleza (Silva-Flores et al., 2005). Las estimaciones se hicieron a los 15, 30, 45 y 60 d después de la aplicación (DDA) de los herbicidas POST, lo que implica alrededor de $25 \mathrm{~d}$ más para los tratamientos PRE. Además se midió la altura promedio de 10 plantas de sorgo por unidad experimental en las mismas fechas de muestreo. El rendimiento de sorgo se obtuvo al cosechar manualmente las panojas de los dos surcos centrales de cada unidad experimental. Las panojas se trillaron y el contenido de humedad del grano se ajustó a $14 \%$. 
Cuadro 2. Escala propuesta por la Sociedad Europea de Investigación en Maleza (EWRS) para evaluar el control de maleza y fitotoxicidad al cultivo por herbicidas.

\begin{tabular}{cclcl}
\hline Valor & $\begin{array}{c}\text { Control } \\
\text { de maleza } \\
(\%)\end{array}$ & \multicolumn{1}{c}{$\begin{array}{c}\text { Efecto sobre } \\
\text { maleza }\end{array}$} & $\begin{array}{c}\text { Fitotoxicidad } \\
\text { al cultivo } \\
(\%)\end{array}$ & $\begin{array}{l}\text { Efecto sobre } \\
\text { el cultivo }\end{array}$ \\
\hline 1 & $99.0-100.0$ & Muerte & $0.0-1.0$ & Sin efecto \\
2 & $96.5-99.0$ & Muy buen control & $1.0-3.5$ & Síntomas muy ligeros \\
3 & $93.0-96.5$ & Buen control & $3.5-7.0$ & Síntomas ligeros \\
4 & $87.5-93.0$ & Control suficiente & $7.0-12.5$ & $\begin{array}{l}\text { Síntomas evidentes sin } \\
\text { efecto en rendimiento }\end{array}$ \\
5 & $80.0-87.5$ & Control medio & $12.5-20.0$ & Daño medio \\
6 & $70.0-80.0$ & Control regular & $20.0-30.0$ & Daño elevado \\
7 & $50.0-70.0$ & Control pobre & $30.0-50.0$ & Daño muy elevado \\
8 & $1.0-50.0$ & Control muy pobre & $50.0-99.0$ & Daño severo \\
9 & $0.0-1.0$ & Sin efecto & $99.0-100.0$ & Muerte \\
\hline
\end{tabular}

Los datos de las variables se sometieron a un análisis de varianza y las medias se compararon con la prueba de Tukey $(\mathrm{P} \leq 0.05)$. El análisis combinado de los datos indicó interacción para la fitotoxicidad y el control de quelite $\mathrm{x}$ años, por lo que estas variables se presentan para cada año por separado. El resto de las variables se presentan como promedios de los dos años. Los datos de fitotoxicidad al cultivo y control de maleza fueron transformados por arcoseno de la raíz cuadrada de $\mathrm{X} / 100$ antes de su análisis, para homogenizar sus varianzas (Lentner y Bishop, 1993), aunque los resultados se presentan con los datos no transformados.

\section{RESULTADOS Y DISCUSIÓN}

\section{Fitotoxicidad 2007}

En las parcelas tratadas con atrazina y amicarbazone aplicados PRE no se observaron síntomas de fitotoxicidad en el sorgo, de los 15 a los 60 DDA (Cuadro 3). Lo anterior demuestra su excelente selectividad al sorgo en suelos arcillosos que poseen mayor adsorción y poca movilidad de estos herbicidas de absorción radical (Baumann et al., 1999; Bachega et al., 2009).

A los 15 DDA se observó fitotoxicidad de prosulfuron de $11 \%$ que causó reducción de la altura del sorgo. Sin embargo, a los 30 y 45 DDA estos síntomas disminuyeron $(\mathrm{P} \leq 0.05)$ y desaparecieron a los $60 \mathrm{DDA}$ (Cuadro 3 ). Prosulfuron es un herbicida del grupo químico de la sulfonilureas que usualmente ocasiona una toxicidad temporal en sorgo. La selectividad de las sulfonilureas en los cultivos tolerantes se basa en su metabolismo en las primeras horas después de su aplicación, por lo que algunas veces se presentan ligeros daños (Brown, 1990). La mezcla de carfentrazone + 2,4-D causó una toxicidad elevada (30 \%) al sorgo a los $15 \mathrm{DDA}$, debido a los efectos de contacto del carfentrazone que ocasionaron manchas necróticas en el follaje del cultivo. Aunque estos síntomas presentaron una tendencia a disminuir con el tiempo, a partir de los 30 DDA se observaron efectos causados por el 2,4-D con una toxicidad elevada (> $20 \%$ ) que se prolongaron hasta los 60 DDA. Según Boydston (2004) y Lyon et al. (2007), la

Cuadro 3. Fitotoxicidad en sorgo con diferentes tratamientos de control de maleza. Río Bravo, Tam. en 2007 y 2008.

\begin{tabular}{|c|c|c|c|c|c|c|c|c|c|}
\hline \multirow{2}{*}{ Tratamiento } & \multirow{2}{*}{$\begin{array}{l}\text { Dosis } \\
\left(\mathrm{g} \mathrm{ha}^{-1}\right)\end{array}$} & \multicolumn{4}{|c|}{ Días después de la aplicación POST (2007) } & \multicolumn{4}{|c|}{ Días después de la aplicación POST (2008) } \\
\hline & & 15 & 30 & 45 & 60 & 15 & 30 & 45 & 60 \\
\hline & & \multicolumn{4}{|c|}{ Fitotoxicidad (\%) } & \multicolumn{4}{|c|}{ Fitotoxicidad (\%) } \\
\hline Amicarbazone & 750 & $0 \mathrm{e}$ & $0 \mathrm{~d}$ & $0 \mathrm{~d}$ & $0 \mathrm{~d}$ & $0 \mathrm{c}$ & $0 \mathrm{~d}$ & $0 \mathrm{c}$ & $0 \mathrm{~d}$ \\
\hline Atrazina & 750 & $0 \mathrm{e}$ & $0 \mathrm{~d}$ & $0 \mathrm{~d}$ & $0 \mathrm{~d}$ & $0 \mathrm{c}$ & $0 \mathrm{~d}$ & $0 \mathrm{c}$ & $0 \mathrm{~d}$ \\
\hline Prosulfuron & 17 & $11 \mathrm{c}$ & $5 \mathrm{c}$ & $7 \mathrm{c}$ & $0 \mathrm{~d}$ & $5 \mathrm{~b}$ & $0 \mathrm{~d}$ & $0 \mathrm{c}$ & $0 \mathrm{~d}$ \\
\hline Carfentrazone $+2,4-\mathrm{D}$ & $2.5+225$ & $30 \mathrm{a}$ & $21 \mathrm{~b}$ & $20 \mathrm{~b}$ & $20 \mathrm{~b}$ & $20 \mathrm{a}$ & $11 \mathrm{c}$ & $14 \mathrm{~b}$ & $17 \mathrm{c}$ \\
\hline 2,4-D & 720 & $16 \mathrm{~b}$ & $40 \mathrm{a}$ & $35 \mathrm{a}$ & $37 \mathrm{a}$ & $19 \mathrm{a}$ & $27 \mathrm{a}$ & $29 \mathrm{a}$ & $30 \mathrm{a}$ \\
\hline Dicamba & 144 & $14 \mathrm{bc}$ & $20 \mathrm{~b}$ & $7 \mathrm{c}$ & $7 \mathrm{c}$ & $16 \mathrm{a}$ & $20 \mathrm{~b}$ & $16 \mathrm{~b}$ & $21 \mathrm{~b}$ \\
\hline Bromoxinil & 480 & $5 \mathrm{~d}$ & $2 \mathrm{~cd}$ & $0 \mathrm{~d}$ & $0 \mathrm{~d}$ & $0 \mathrm{c}$ & $0 \mathrm{~d}$ & $0 \mathrm{c}$ & $0 \mathrm{~d}$ \\
\hline Bentazona & 960 & $4 \mathrm{de}$ & $4 \mathrm{c}$ & $0 \mathrm{~d}$ & $0 \mathrm{~d}$ & $0 \mathrm{c}$ & $0 \mathrm{~d}$ & $0 \mathrm{c}$ & $0 \mathrm{~d}$ \\
\hline Testigo con maleza & & $0 \mathrm{e}$ & $0 \mathrm{~d}$ & $0 \mathrm{~d}$ & $0 \mathrm{~d}$ & $9 \mathrm{c}$ & $0 \mathrm{~d}$ & $0 \mathrm{c}$ & $0 \mathrm{~d}$ \\
\hline Testigo limpio & & $0 \mathrm{e}$ & $0 \mathrm{~d}$ & $0 \mathrm{~d}$ & $0 \mathrm{~d}$ & $0 \mathrm{c}$ & $0 \mathrm{~d}$ & $0 \mathrm{c}$ & $0 \mathrm{~d}$ \\
\hline
\end{tabular}

Medias con letras iguales en cada columna no son estadísticamente diferentes (Tukey, 0.05). 
mezclas de carfentrazone + dicamba o 2,4-D causan daños similares en maíz (Zea mays L.) y mijo (Panicum miliaceum L.) con recuperación de plantas al emitir las hojas nuevas sin daños.

Los herbicidas 2,4-D y dicamba presentaron daños medios en el sorgo a los 15 DDA (Cuadro 3), caracterizados por la curvatura de las plantas y malformaciones de sus hojas, daños típicos de herbicidas hormonales (Baumann et al., 1999). A los 30 DDA los daños de 2,4-D se incrementaron a $40 \%$ y fueron superiores $(\mathrm{P} \leq 0.05)$ a los causados por el dicamba. Además, los daños causados por el 2,4-D se mantuvieron a los 45 y 60 DDA mientras que los de dicamba se redujeron $(\mathrm{P} \leq 0.05)$ se ha demostrado que el $2,4-\mathrm{D}$ presenta mayor toxicidad que el dicamba en cultivos sensibles (Sciumbato et al., 2004).

Los herbicidas de contacto bromoxinil y bentazona causaron daños ligeros al sorgo a los 15 y 30 DDA, caracterizados por manchas necróticas en los ápices de las hojas tratadas. Sin embargo, estos daños no se reflejaron en las hojas nuevas del cultivo a los 45 y 60 DDA. Bromoxinil y bentazona se han reportado como herbicidas altamente selectivos al sorgo (Regehr et al., 2011).

\section{Fitotoxicidad 2008}

En general los daños causados al sorgo en 2008 fueron inferiores a los observados en 2007 debido a un menor contenido de humedad en el suelo en los días siguientes a la aplicación, lo que hizo menos activos a los herbicidas PRE y POST (datos no mostrados). Al igual que en 2007, no se observaron daños al sorgo por la aplicación PRE de atrazina y amicarbazone (Cuadro 3). Los daños causados por prosulfuron al sorgo a los 15 DDA fueron ligeros e inferiores a los observados en 2007 y desaparecieron a partir de los 30 DDA. La mezcla de carfentrazone + 2,4-D nuevamente dañó al cultivo a los $15 \mathrm{DDA}$, pero a partir de los 30 DDA los síntomas disminuyeron. Por su parte, 2,4-D causó daños superiores en 27 a $30 \%$ a los causados por el dicamba en el sorgo de los 30 a los $60 \mathrm{DDA}(\mathrm{P} \leq 0.05)$. No se observaron daños por bromoxinil o bentazona (Cuadro 3 ).

\section{Control de polocote}

Hubo un control suficiente (92 \%) de polocote con amicarbazone a los 15 DDA (Cuadro 4), equivalente a 40 $\mathrm{d}$ después de la emergencia del sorgo, que cubre el periodo crítico de competencia maleza - cultivo. Este control se mantuvo igual a los 30 DDA y disminuyó ligeramente a los 45 y 60 DDA, lo que manifiesta un control residual de este herbicida. Atrazina mostró un comportamiento similar ( $\mathrm{P}$ $\leq 0.05)$ al amicarbazone con un control medio de polocote en los primeros 30 DDA (80 a $87 \%$ ), y con una ligera disminución después de ese periodo.

La maleza polocote resultó altamente susceptible a la acción del prosulfuron ya que la eliminó en un porcentaje mayor a $94 \%$ de los 15 a los 60 DDA (Cuadro 4). En estudios anteriores se observó que esta maleza y otras de la familia Asteraceae, como Parthenium hysterophorus y Xanthium strumarium, son fácilmente controladas con prosulfuron (Ma et al., 1997; Rosales-Robles et al., 2005b). La mezcla de carfentrazone $+2,4$-D mostró un control medio a suficiente (84 a $89 \%$ ) de polocote desde los 15 a los 45 DDA, con una ligera disminución a los $60 \mathrm{DDA}$. El carfentrazone por ser un herbicida de contacto, incrementó en alrededor de $10 \%$ la acción del 2,4-D a los 15 DDA. El 2,4-D requirió de 30 DDA para mostrar un control suficiente de polocote, control que se extendió hasta los 60 DDA. El otro herbicida de acción hormonal (dicamba) tuvo un comportamiento similar al 2,4-D (Cuadro 4).

Bromoxinil también mostró un buen control de polocote (>92\%) de los 15 a los 60 DDA, al eliminar eficientemente la primera emergencia de esta maleza que es la más

Cuadro 4. Control de Heliantus annuus L. en sorgo con diferentes tratamientos de control de maleza. Río Bravo, Tam. (promedio de dos años).

\begin{tabular}{lccccc}
\hline \multirow{2}{*}{ Tratamiento } & \multirow{2}{*}{$\begin{array}{c}\text { Dosis } \\
\left(\mathrm{g} \mathrm{ha}^{-1}\right)\end{array}$} & \multicolumn{4}{c}{ Días después de la aplicación POST } \\
\cline { 3 - 6 } & & 15 & 30 & 45 & 60 \\
\cline { 3 - 6 } Amicarbazone & 750 & $92 \mathrm{ab}$ & $87 \mathrm{abc}$ & $82 \mathrm{~cd}$ & $85 \mathrm{~cd}$ \\
Atrazina & 750 & $87 \mathrm{bc}$ & $80 \mathrm{c}$ & $79 \mathrm{~d}$ & $77 \mathrm{~d}$ \\
Prosulfuron & 17 & $95 \mathrm{ab}$ & $96 \mathrm{ab}$ & $97 \mathrm{a}$ & $97 \mathrm{ab}$ \\
Carfentrazone +2,4-D & $2.5+225$ & $86 \mathrm{bcd}$ & $84 \mathrm{bc}$ & $89 \mathrm{abcd}$ & $77 \mathrm{~d}$ \\
2,4-D & 720 & $77 \mathrm{~cd}$ & $92 \mathrm{abc}$ & $94 \mathrm{ab}$ & $90 \mathrm{abc}$ \\
Dicamba & 144 & $76 \mathrm{~d}$ & $79 \mathrm{c}$ & $92 \mathrm{abc}$ & $89 \mathrm{bc}$ \\
Bromoxinil & 480 & $97 \mathrm{a}$ & $95 \mathrm{ab}$ & $94 \mathrm{ab}$ & $92 \mathrm{abc}$ \\
Bentazona & 960 & $85 \mathrm{bcd}$ & $79 \mathrm{c}$ & $85 \mathrm{bcd}$ & $76 \mathrm{~d}$ \\
Testigo con maleza & & $0 \mathrm{e}$ & $0 \mathrm{~d}$ & $0 \mathrm{e}$ & $0 \mathrm{e}$ \\
Testigo limpio & & $100 \mathrm{a}$ & $100 \mathrm{a}$ & $100 \mathrm{a}$ & $100 \mathrm{a}$ \\
\hline
\end{tabular}

Medias con letras iguales en cada columna no son estadísticamente diferentes (Tukey, 0.05). 
importante en el sorgo (Acosta y Agundis, 1976), lo que confirma la alta efectividad de este herbicida en el control de la maleza (Rosales-Robles et al., 2005b). El control de polocote con bentazona fue inferior $(\mathrm{P} \leq 0.05)$ al obtenido con bromoxinil en todas las fechas de muestreo, lo que demuestra una menor susceptibilidad de esta maleza a bentazona (Cuadro 4).

\section{Control de quelite 2007}

A los 15 DDA se logró un control suficiente del quelite con amicarbazone y atrazina (92 a $94 \%$ ) aplicados PRE, sin diferencias entre ambos (Cuadro 5). Este periodo incluye los primeros $40 \mathrm{~d}$ de desarrollo del sorgo, ya que estos herbicidas se aplicaron $1 \mathrm{~d}$ después de la siembra. A partir de los 30 DDA se observó una disminución ligera en la acción de ambos herbicidas, especialmente a los 60 DDA, pero el control de esta especie fue medio.
El quelite se logró controlar en $92 \%$ a los 15 DDA tanto con amicarbazone como con atrazina, aunque a partir de los 30 DDA disminuyó a 82 \% (Cuadro 7). En 2008 con prosulfuron sólo se logró un control medio (80 \%) del quelite de los 15 a los $60 \mathrm{DDA}$, probablemente por una disminución en la humedad en el suelo (datos no mostrados) y un mayor desarrollo del quelite. La aplicación de $2,4-\mathrm{D}$, solo o en mezcla con carfetrazone resultó ser una opción adecuada en el control de quelite a partir de los 30 DDA. El dicamba presentó resultados similares. (Cuadro 6).

Bromoxinil superó $(P \leq 0.05)$ a bentazona en el control de quelite a los $30 \mathrm{DDA}$, pero a partir de los $45 \mathrm{DDA}$ ambos herbicidas fueron similares, con un control regular de esta maleza. Fausey y Renner (2001) reportaron una eficiencia baja de bentazona en el control de quelite, aún por debajo de los niveles observados en este trabajo, probablemente por diferencias en las condiciones ambientales, principalmente

Cuadro 5. Control de Amaranthus palmeri S. Wats. en sorgo con diferentes tratamientos de control de maleza. Río Bravo, Tam. en 2007 y 2008.

\begin{tabular}{|c|c|c|c|c|c|c|c|c|c|}
\hline \multirow{2}{*}{ Tratamiento } & \multirow{2}{*}{$\begin{array}{l}\text { Dosis } \\
\left(\mathrm{g} \mathrm{ha}^{-1}\right)\end{array}$} & \multicolumn{4}{|c|}{ Días después de la aplicación POST (2007) } & \multicolumn{4}{|c|}{ Días después de la aplicación POST (2008) } \\
\hline & & 15 & 30 & 45 & 60 & 15 & 30 & 45 & 60 \\
\hline & & \multicolumn{4}{|c|}{ Control (\%) } & \multicolumn{4}{|c|}{ Control (\%) } \\
\hline Amicarbazone & 750 & $92 \mathrm{ab}$ & $90 \mathrm{bcd}$ & $86 \mathrm{bcd}$ & $82 \mathrm{c}$ & $92 \mathrm{ab}$ & $82 \mathrm{bc}$ & $80 \mathrm{bcd}$ & $80 \mathrm{bc}$ \\
\hline Atrazina & 750 & $94 \mathrm{ab}$ & $87 \mathrm{~cd}$ & $85 \mathrm{~cd}$ & $85 \mathrm{bc}$ & $92 \mathrm{ab}$ & $87 \mathrm{ab}$ & $82 \mathrm{bcd}$ & $82 \mathrm{bc}$ \\
\hline Prosulfuron & 17 & $97 \mathrm{a}$ & $94 \mathrm{abc}$ & $95 \mathrm{ab}$ & $92 \mathrm{ab}$ & $80 \mathrm{~cd}$ & $80 \mathrm{bc}$ & $82 \mathrm{bcd}$ & $80 \mathrm{bc}$ \\
\hline Carfentrazone $+2,4-\mathrm{D}$ & $2.5+225$ & $90 \mathrm{abc}$ & $95 \mathrm{abc}$ & $93 a b c$ & $95 \mathrm{a}$ & $89 \mathrm{bc}$ & $89 \mathrm{ab}$ & $88 \mathrm{bc}$ & $87 \mathrm{ab}$ \\
\hline 2,4-D & 720 & $80 \mathrm{bc}$ & $94 \mathrm{abc}$ & $97 \mathrm{a}$ & 98 a & $80 \mathrm{~cd}$ & $89 \mathrm{ab}$ & $90 \mathrm{ab}$ & $90 \mathrm{ab}$ \\
\hline Dicamba & 144 & $74 \mathrm{c}$ & $96 \mathrm{ab}$ & 95 a & 96 a & $82 \mathrm{~cd}$ & $82 \mathrm{bc}$ & $87 \mathrm{bc}$ & $87 \mathrm{ab}$ \\
\hline Bromoxinil & 480 & $96 \mathrm{ab}$ & $97 \mathrm{ab}$ & $96 \mathrm{a}$ & 96 a & $85 \mathrm{bcd}$ & $86 \mathrm{~b}$ & $77 \mathrm{~cd}$ & $80 \mathrm{bc}$ \\
\hline Bentazona & 960 & $88 \mathrm{abc}$ & $85 \mathrm{~d}$ & $82 \mathrm{~d}$ & $77 \mathrm{c}$ & $77 \mathrm{~d}$ & $70 \mathrm{c}$ & $72 \mathrm{~d}$ & $72 \mathrm{c}$ \\
\hline Testigo con maleza & & $0 \mathrm{~d}$ & $0 \mathrm{e}$ & $0 \mathrm{e}$ & $0 \mathrm{e}$ & $0 \mathrm{e}$ & $0 \mathrm{~d}$ & $0 \mathrm{e}$ & $0 \mathrm{~d}$ \\
\hline Testigo limpio & & $100 \mathrm{a}$ & $100 \mathrm{a}$ & $100 \mathrm{a}$ & $100 \mathrm{a}$ & $100 \mathrm{a}$ & $100 \mathrm{a}$ & $100 \mathrm{a}$ & $100 \mathrm{a}$ \\
\hline
\end{tabular}

Medias con letras iguales en cada columna no son estadísticamente diferentes (Tukey, 0.05)

El prosulfuron eliminó al quelite con una eficiencia del 94 a $97 \%$ a los 15 y 30 DDA, el efecto que se mantuvo a los 45 y 60 DDA (Cuadro 5). En estudios similares se ha demostrado que prosulfuron tiene un control aceptable de quelite y otras malezas de la familia Amaranthaceae (Grichar et al., 2000). La mezcla de carfentrazone + 2,4-D controló al quelite en un porcentaje superior a $90 \%$ de los 15 a los 60 DDA. La mezcla de carfentrazone con 2,4-D evidenció un mayor control de esta maleza a los 15 DDA. También 2,4-D y dicamba mostraron un buen control del quelite a partir de los $30 \mathrm{DDA}$, sin diferencias entre ambos herbicidas (Cuadro 5). Bromoxinil mostró un buen control de quelite y superó $(\mathrm{P} \leq 0.05)$ a bentazona cuyo control fue sólo medio. humedad de suelo y humedad relativa, que afectan a los herbicidas con acción de contacto.

\section{Control de trompillo}

Trompillo es una maleza perenne con reproducción vegetativa por rizomas que fue difícil de controlar con los herbicidas evaluados. No se observaron efectos significativos con la aplicación en PRE de los herbicidas amicarbazone y atrazina (Cuadro 6). Prosulfuron causó sólo efectos muy ligeros $(\leq 12 \%)$ en todas las fechas de muestreo. En otros estudios se ha observado que algunos herbicidas de la familia sulfonilureas, como prosulfuron, no controlan de manera eficiente a las malezas de la familia Solanaceae, como trompillo, Solanum americanum (Carey et al., 1997) y S. carolinense (Prostko et al., 1994). Al parecer la tolerancia de esta familia de malezas se debe al transporte

\section{Control de quelite 2008}


Cuadro 6. Control de Solanum elaeagnifolium Cav. en sorgo con diferentes tratamientos de control de maleza. Río Bravo, Tama. (promedio de dos años).

\begin{tabular}{lcrrrr}
\hline \multirow{2}{*}{ Tratamiento } & $\begin{array}{c}\text { Dosis } \\
\left(\mathrm{g} \mathrm{ha}^{-1}\right)\end{array}$ & \multicolumn{5}{c}{ Días después de la aplicación POST } \\
& & \multicolumn{5}{c}{ Control (\%) } \\
\cline { 3 - 6 } Amicarbazone & 750 & $0 \mathrm{~d}$ & $4 \mathrm{de}$ & $4 \mathrm{de}$ & $0 \mathrm{~d}$ \\
Atrazina & 750 & $0 \mathrm{~d}$ & $7 \mathrm{de}$ & $7 \mathrm{de}$ & $0 \mathrm{~d}$ \\
Prosulfuron & 17 & $7 \mathrm{~d}$ & $10 \mathrm{de}$ & $12 \mathrm{de}$ & $2 \mathrm{~d}$ \\
Carfentrazone +2,4-D & $2.5+225$ & $74 \mathrm{~b}$ & $79 \mathrm{bc}$ & $80 \mathrm{bc}$ & $85 \mathrm{bc}$ \\
2,4-D & 720 & $74 \mathrm{~b}$ & $85 \mathrm{~b}$ & $85 \mathrm{~b}$ & $90 \mathrm{~b}$ \\
Dicamba & 144 & $66 \mathrm{~b}$ & $70 \mathrm{c}$ & $73 \mathrm{c}$ & $82 \mathrm{c}$ \\
Bromoxinil & 480 & $29 \mathrm{c}$ & $15 \mathrm{~d}$ & $12 \mathrm{de}$ & $5 \mathrm{~d}$ \\
Bentazona & 960 & $29 \mathrm{c}$ & $5 \mathrm{de}$ & $5 \mathrm{de}$ & $2 \mathrm{~d}$ \\
Testigo con maleza & & $0 \mathrm{~d}$ & $0 \mathrm{e}$ & $0 \mathrm{e}$ & $0 \mathrm{~d}$ \\
Testigo limpio & & $100 \mathrm{a}$ & $100 \mathrm{a}$ & $100 \mathrm{a}$ & $100 \mathrm{a}$ \\
\hline
\end{tabular}

Medias con letras iguales en cada columna no son estadísticamente diferentes (Tukey, 0.05)

limitado y al metabolismo del herbicida (Carey et al., 1997; Buker et al., 2004).

El mayor control de trompillo (79 a 90 \%) se logró a partir de los 30 DDA con 2,4-D solo o en mezcla con carfentrazone, sin diferencias significativas entre estos tratamientos (Cuadro 6). Lyon et al. (2007) reportaron resultados similares en el control de Solanum rostratum, con la mezcla de carfentrazone $+2,4-\mathrm{D}$. Dicamba controló el trompillo en menor grado que el 2,4-D de los 30 a los 60 DDA. Los herbicidas hormonales POST se consideran los tratamientos más eficientes en el control de esta maleza trompillo (Eleftherohorinos et al., 1993). Los herbicidas de contacto bromoxinil y bentazona sólo presentaron efectos temporales en el follaje del trompillo, pues la maleza se recuperó a partir de los 30 DDA (Cuadro 6). Los herbicidas de contacto comúnmente resultan en un control ineficiente de malezas perennes con reproducción vegetativa.

\section{Altura del sorgo}

La aplicación PRE de amicarbazone y atrazina, y la aplicación POST de dicamba, bromoxinil y bentazona no afectaron la altura del sorgo (Cuadro 7). Prosulfuron causó una ligera disminución de parte del sorgo a los 15 DDA, pero este efecto se revirtió a partir de los 30 DDA. Es común que este herbicida cause ligeros daños al sorgo en las primeras dos semanas después de su aplicación (RosalesRobles et al., 2005b), efecto que es temporal de manera que el sorgo se recupera en pocos días.

El 2,4-D solo y en mezcla con carfentrazone redujo ( $\mathrm{P}$ $\leq 0.05$ ) la altura del sorgo (Cuadro 7). Sin embargo, con el 2,4-D aplicado a $720 \mathrm{~g} \mathrm{ha}^{-1}$ la reducción se mantuvo hasta

\begin{tabular}{|c|c|c|c|c|c|c|}
\hline \multirow{3}{*}{ Tratamiento } & \multirow{3}{*}{$\begin{array}{l}\text { Dosis } \\
\left(\mathrm{g} \mathrm{ha}^{-1}\right)\end{array}$} & \multicolumn{4}{|c|}{ Días después de la aplicación POST } & \multirow{3}{*}{$\begin{array}{l}\text { Rendimiento } \\
\left(\mathrm{kg} \mathrm{ha}^{-1}\right)\end{array}$} \\
\hline & & 15 & 30 & 45 & 60 & \\
\hline & & \multicolumn{4}{|c|}{ Altura $(\mathrm{cm})$} & \\
\hline Amicarbazone & 750 & $65 \mathrm{ab}$ & $95 \mathrm{ab}$ & $119 \mathrm{ab}$ & $124 \mathrm{ab}$ & $5690 \mathrm{ab}$ \\
\hline Atrazina & 750 & $66 \mathrm{ab}$ & $97 \mathrm{ab}$ & $120 \mathrm{ab}$ & $125 \mathrm{ab}$ & 5920 a \\
\hline Prosulfuron & 17 & $63 \mathrm{bc}$ & $93 \mathrm{abc}$ & $122 \mathrm{ab}$ & $126 \mathrm{ab}$ & 6030 a \\
\hline Carfentrazone $+2,4-\mathrm{D}$ & $2.5+225$ & $63 \mathrm{bc}$ & 87 bcd & $117 \mathrm{~b}$ & $121 \mathrm{~b}$ & $4870 \mathrm{c}$ \\
\hline $2,4-\mathrm{D}$ & 720 & $60 \mathrm{c}$ & $84 \mathrm{~cd}$ & $110 \mathrm{c}$ & $113 \mathrm{c}$ & $4950 \mathrm{bc}$ \\
\hline Dicamba & 144 & $67 \mathrm{a}$ & $91 \mathrm{abc}$ & $121 \mathrm{ab}$ & $122 \mathrm{ab}$ & $5390 \mathrm{abc}$ \\
\hline Bromoxinil & 480 & $67 \mathrm{a}$ & $95 \mathrm{ab}$ & $125 \mathrm{a}$ & $128 \mathrm{a}$ & $5730 \mathrm{ab}$ \\
\hline Bentazona & 960 & $63 \mathrm{ab}$ & $91 \mathrm{abc}$ & $121 \mathrm{ab}$ & $123 \mathrm{ab}$ & $5310 \mathrm{abc}$ \\
\hline Testigo con maleza & & $64 \mathrm{ab}$ & $82 \mathrm{~d}$ & $109 \mathrm{c}$ & $107 \mathrm{c}$ & $2115 \mathrm{~d}$ \\
\hline Testigo limpio & & $65 \mathrm{ab}$ & $95 \mathrm{ab}$ & $123 \mathrm{ab}$ & $127 \mathrm{ab}$ & $5960 \mathrm{a}$ \\
\hline
\end{tabular}

Medias con letras iguales en cada columna no son estadísticamente diferentes (Tukey, 0.05) 
los 60 DDA, mientras el sorgo tratado con la mezcla de 2,4$\mathrm{D}$ a $225 \mathrm{~g} \mathrm{ha}^{-1}$ con carfentrazone, se recuperó a partir de los 30 DDA. La reducción de la altura del sorgo se debió a la curvatura y malformación de sus tallos, síntomas típicos de herbicidas hormonales (Regehr et al., 2011).

La competencia de la maleza con el cultivo se reflejó en una reducción $(\mathrm{P} \leq 0.05)$ de la altura del sorgo en el testigo con maleza a partir de los $30 \mathrm{DDA}$, reducción que fue más evidente a los 45 y 60 DDA. El porte del testigo limpio superó en 12 a $20 \mathrm{~cm}$ al testigo con maleza de los 30 a los 60 DDA.

\section{Rendimiento de sorgo}

La aplicación de los herbicidas atrazina, amicarbazone, prosulfuron, dicamba, bromoxinil y bentazona resultó en un rendimiento de grano similar al del testigo limpio, de $5960 \mathrm{~kg} \mathrm{ha}^{-1}$ (Cuadro 7). Los tratamientos de 2,4-D y su mezcla con carfentrazone presentaron una reducción del rendimiento de $17 \%(\mathrm{P} \leq 0.05)$ con relación al del testigo limpio. Lo anterior fue como consecuencia de los altos niveles de fitotoxicidad durante el desarrollo del cultivo. Cabe señalar que los daños causados por dicamba en los primeros $30 \mathrm{DDA}$ no afectaron el rendimiento del sorgo. El testigo con maleza presentó una reducción del rendimiento de $65 \%$ debido a la competencia de la maleza con el cultivo por luz, agua y nutrimentos.

\section{CONCLUSIONES}

Los herbicidas atrazina y amicarbazone aplicados en preemergencia mostraron un control suficiente de polocote y quelite sin causar daños al sorgo, pero no controlaron eficientemente al trompillo. Los herbicidas aplicados en post-emergencia mostraron un control eficiente de polocote, la principal maleza del norte de Tamaulipas. El control suficiente de quelite y trompillo sólo fue obtenido en ambos años con 2,4-D sólo o en mezcla con carfentrazone y con dicamba; sin embargo, estos herbicidas causaron daños al sorgo que redujeron su rendimiento, con excepción de dicamba. Ninguno de los herbicidas fue efectivo contra las tres especies de malezas evaluadas, sin causar daños al sorgo.

\section{AGRADECIMIENTOS}

Los autores agradecen a la Fundación Produce Tamaulipas, A.C. el apoyo financiero de este trabajo, y a Esteban Robles Escarriola por su apoyo técnico en la conducción de la investigación.

\section{BIBLIOGRAFÍA}

Acosta S, O Agundis (1976) Época de emergencia de las principales malas hierbas de la región norte de Tamaulipas. Agric. Téc. Méx. III:437441.

Bachega T F, M D Pavani, P L Alves, L P Saes, M Boschiero (2009) Leaching of sulfentrazone and amicarbazon in soil columns with mineral oil. Planta Daninha 27:363-370.

Baumann P A, P A Dotray, E P Prostko (1999) Herbicides: how they work and the symptoms they cause. Texas Agricultural Extension Service. Texas A\&M University. B-6081. 12 p.

Boydston R A (2004) Managing volunteer potato (Solanum tuberosum) in field corn (Zea mays) with carfentrazone-ethyl and dicamba. Weed Technol. 18:83-87.

Brown H M (1990) Mode of action, crop selectivity, and soil relations of the sulfonylurea. Pesticide Sci. 29:263-281.

Buker R S, B Rathinasapathi, W M Stall, G MacDonald, S M Olson (2004) Physiological basis for differential tolerance of tomato and pepper to rimsulfuron and halosulfuron: site of action study. Weed Sci. 52:201-205.

Carey J D, D Penner, J J Kells (1997) Physiological basis for nicosulfuron and primisulfuron selectivity in five plant species. Weed Sci. 45:2230.

Eleftherohorinos I G, C E Bell, E Kotoula-Syka (1993) Silverleaf nightshade (Solanum elaeagnifolium) control with foliar herbicides. Weed Technol. 7:808-811.

Fausey J C, K A Renner (2001) Broadleaf weed control in corn (Zea mays) and soybean (Glycine max) with CGA-248757 and flumiclorac alone and in tank mixtures. Weed Technol. 15:399-407.

Finaciera Rural, Dirección General Adjunta de Planeación Estratégica y Análisis Sectorial (2009) Monografía del sorgo. Disponible en: http://www.financierarural.gob.mx/informacionsectorrural/ Documents/MONOGRAFIA\%20SORGO-septiembre.pdf (Enero 2011).

Grichar W J, D C Sestak, K Brewer, B Minton (2000) Weed control with CGA 152005 and peanut (Arachis ipogea) response. Weed Technology 14: 218-222.

Lentner M, T Bishop (1993) Experimental Design and Analysis. Valley Book Co. Blacksburg, VA. USA. 585 p.

Lyon D J, A Kniss, S D Miller (2007) Carfentrazone improves broadleaf weed control in proso and fox millets. Weed Technol. 21:84-87.

Ma G, H D Coble, F T Corbin, J D Burton (1997) Physiological mechanisms for differential responses of three weed species to prosulfuron. Weed Sci. 45:642-647.

O'Sullivan J, R J Thomas (2001) Injury and yield effects of crops grown in CGA-152005 treated soil. Weed Technol. 15:594-597.

Prostko E P, J Ingerson-Mahar, B A Majek (1994) Postemergence horsenettle (Solanum carolinense) control in field corn (Zea mays). Weed Technol. 8:441-444.

Regher D L, D E Peterson, W H Fick, P W Stahlman, R E Wolf (2011) Chemical Weed Control for Field Crops, Pastures, Rangeland, and Noncropland. Report of Progress 1045. Manhattan, KS, Kansas State Univ. 123 p.

Rosales-Robles E, R Sánchez-de la Cruz, J Salinas-García (2005a) Período crítico de competencia del polocote (Helianthus annuus) en sorgo para grano. Agrociencia 39: 205-210.

Rosales-Robles E, R Sánchez-de la Cruz, J Salinas-García, V PecinaQuintero (2005b) Broadleaf weed management in grain sorghum with reduced rates of postemergence herbicides. Weed Technol. 19: 385-290.

SAGARPA, Secretaría de Agricultura, Ganadería, Desarrollo Rural, Pesca y Alimentación (2011) SIAP, Sistema de Información Agroalimentaria y Pesquera. Disponible en: http://www.siap. sagarpa.gob.mx/ (Enero 2011).

Sciumbato A S, J M Chandler, S A Senseman, R W Bovey, K L Smith (2004) Determining exposure to auxin-like herbicides. II. Practical application to quantify volatility. Weed Technol. 18:1135-1142.

Silva-Flores M A, J C Rodríguez-Maciel, O Díaz-Gómez, N BautistaMartínez (2005) Efectividad biológica de un derivado de ácido graso para el control de Macrosiphum rosae L. (Homoptera: Aphididae) y Tetranychus urticae Koch (Acari: Tetranychidae). Agrociencia 39:319-325.

Wicks G A, A R Martin, A E Haack, G W Mahnken (1994) Control of triazine-resistant kochia (Kochia scoparia) in sorghum (Sorghum bicolor). Weed Technol. 8:748-753. 